\title{
A "strategy tripod" perspective on export behaviors: Evidence from domestic and foreign firms based in an emerging economy
}

Gerald Yong Gao, Janet Y Murray, Masaaki Kotabe and jiangyong Lu
Journal of International Business Studies (2010) 41, |090-109|.

doi: $10.1057 / j i b s .2010 .32$

Correction to: Journal of International Business Studies (2010) 41, 377-396. doi: 10.1057/jibs.2009.27

Owing to a production error, a number of values were incorrectly shown in Table 6 of this article. A corrected version of this table is given overleaf. 
Table 6 The effects of export propensity and export intensity on firm performance: Subgroup analysis

\begin{tabular}{|c|c|c|c|c|c|c|c|c|}
\hline & \multirow[b]{2}{*}{ All firms } & \multirow{2}{*}{\multicolumn{2}{|c|}{ Domestic private enterprises }} & \multirow[b]{2}{*}{$\begin{array}{c}\text { Foreign wholly owned } \\
\text { subsidiaries }\end{array}$} & \multicolumn{4}{|c|}{ With correction for self-selection $(\lambda)$} \\
\hline & & & & & All firms & \multicolumn{2}{|c|}{$\begin{array}{c}\text { Domestic private } \\
\text { enterprises }\end{array}$} & $\begin{array}{c}\text { Foreign } \\
\text { wholly owned } \\
\text { subsidiaries }\end{array}$ \\
\hline \multicolumn{9}{|c|}{ Effects on performance (ROS) } \\
\hline Export propensity & 0.001 & \multirow{2}{*}{\multicolumn{2}{|c|}{$\begin{array}{l}-0.004^{\star * *} \\
-0.014^{\star * \star}\end{array}$}} & $-0.013^{* * *}$ & $-0.002^{*}$ & \multicolumn{2}{|c|}{$-0.010^{\star * *}$} & $-0.013^{\star * *}$ \\
\hline \multirow[t]{3}{*}{ Export intensity } & $-0.021^{* * *}$ & & & $-0.032^{\star * *}$ & $-0.021^{* * *}$ & \multicolumn{2}{|c|}{$-0.011^{\star * *}$} & $-0.029^{\star \star *}$ \\
\hline & & & & & \multicolumn{4}{|c|}{ With correction for self-selection $(\lambda)$} \\
\hline & $\begin{array}{l}\text { Pure cost } \\
\text { leadership } \\
\text { group }\end{array}$ & Pure differentiation group & Hybrid group & $\begin{array}{c}\text { Unattractive } \\
\text { combination group }\end{array}$ & $\begin{array}{l}\text { Pure cost } \\
\text { leadership } \\
\text { group }\end{array}$ & $\begin{array}{c}\text { Pure } \\
\text { differentiation } \\
\text { group }\end{array}$ & $\begin{array}{l}\text { Hybrid } \\
\text { group }\end{array}$ & $\begin{array}{c}\text { Unattractive } \\
\text { combination } \\
\text { group }\end{array}$ \\
\hline \multicolumn{9}{|c|}{ Effects on performance (ROS) for domestic private enterprises by competencies groups } \\
\hline Export propensity & $-0.003^{* * *}$ & 0.005 & $0.005^{* *}$ & $-0.001^{*}$ & $-0.007^{* * *}$ & 0.014 & 0.002 & $-0.011^{\star *}$ \\
\hline \multirow[t]{3}{*}{ Export intensity } & $-0.008^{* \star *}$ & -0.007 & -0.006 & $-0.020^{\star * *}$ & $-0.007^{\star \star *}$ & -0.011 & -0.004 & $-0.017^{\star \star *}$ \\
\hline & & & & & \multicolumn{4}{|c|}{ With correction for self-selection $(\lambda)$} \\
\hline & $\begin{array}{l}\text { Pure cost } \\
\text { leadership } \\
\text { group }\end{array}$ & Pure differentiation group & Hybrid group & $\begin{array}{l}\text { Unattractive } \\
\text { combination group }\end{array}$ & $\begin{array}{l}\text { Pure cost } \\
\text { leadership } \\
\text { group }\end{array}$ & $\begin{array}{c}\text { Pure } \\
\text { differentiation } \\
\text { group }\end{array}$ & $\begin{array}{l}\text { Hybrid } \\
\text { group }\end{array}$ & $\begin{array}{c}\text { Unattractive } \\
\text { combination } \\
\text { group }\end{array}$ \\
\hline \multicolumn{9}{|c|}{ Effects on performance (ROS) for foreign wholly owned subsidiaries by competencies groups } \\
\hline Export propensity & $-0.003^{* *}$ & 0.003 & -0.001 & $0.008^{* *}$ & $-0.009^{* * *}$ & 0.004 & -0.004 & -0.005 \\
\hline Export intensity & $-0.015^{\star * *}$ & 0.007 & -0.002 & $-0.029^{\star * *}$ & $-0.014^{* \star *}$ & 0.001 & -0.003 & $-0.027^{* *}$ \\
\hline
\end{tabular}

${ }^{* * *} \mathrm{p}<0.001,{ }^{* *} \mathrm{p}<0.01,{ }^{*} \mathrm{p}<0.05$

The effects of export propensity and export intensity on Return on Sales (ROS) were estimated by the model:

$\operatorname{ROS}_{i t}=\alpha+\beta_{1} X_{i, t}+\beta_{2}$ FirmSize $_{i, t}$

$+\beta_{3}$ IndustryGrowth $_{i, t-1}+\delta D_{s, t}+\varepsilon_{i t}$

where $X_{i, t}$ is the firm's export propensity or export intensity and $D_{s, t}$ is a vector of industry and year fixed effects. Correction for self-selection $(\lambda)$ was included in the two-stage models. 